\title{
8 Modell-Auswahl für Politik- und Verwaltungsentscheidungen am Beispiel der Pocken-Epidemiologie ${ }^{1}$
}

Zusammenfassung: Öffentliche Entscheidungsträger verwenden zunehmend wissenschaftliche Methoden, einschließlich Simulationsmodellen, um ihre Entscheidungen zu rechtfertigen. Wissenschaftler, Verwaltungsfachleute und Politiker stehen dabei vor einer großen Auswahl an möglichen Modellierungsstrategien. In diesem Vortrag skizziere ich einige Kriterien, die helfen sollen, eine solche Auswahl zu systematisieren. Am Beispiel der Pockenepidemiologie werden drei Arten von Strategien unterschieden: Massive Simulationsmodelle (MSMs), Abstrakte Simulationsmodelle (ASMs) und Makro-Gleichungsmodelle (MEMs). Durch die Analyse einiger der wichtigsten Pocken-Epidemiemodelle, die in den letzten zwanzig Jahren vorgeschlagen wurden, wird erörtert, wie die Strategiewahl mit Bezug auf die Kerncharakteristika dieser jeweiligen Strategien gerechtfertigt werden kann. Erstens argumentiere ich, dass MEMs oft für öffentliche Entscheidungen ausreichen und nur ersetzt werden müssen, wenn sie nicht ausreichend robust sind. Eine solche Robustheitsanalyse erfordert jedoch nur die Verwendung von ASMs, nicht von MSMs. Zweitens argumentiere ich, dass MSM in verschiedenen Dimensionen zwar größeres Potenzial haben als ASMs, sie aber auch eher scheitern - und dass in vielen Fällen diese Wahrscheinlichkeit des Scheiterns ihr höheres Potenzial überwiegt. Zu diesen Dimensionen gehören insbesondere die Repräsentation des relevanten Ziels, die genaue Messung der relevanten Parameter, die Anzahl der enthaltenen Parameter, die Anzahl der simultan modellierten Mechanismen und die Art und Weise, mit struktureller Unsicherheit umzugehen. Während dies keineswegs die Möglichkeit ausschließt, dass einige MSM gute Begründungen für politische Entscheidungen liefern, will ich mit meinen Argumenten vor einer generellen Bevorzugung von MSM gegenüber ASMs für politische Entscheidungszwecke im Allgemeinen und Impfprobleme im Besonderen warnen.

Abstract: Policymakers increasingly draw on scientific methods, including simulation modeling, to justify their decisions. For these purposes, scientists and policymakers face an extensive choice of modeling strategies. Discussing the example of smallpox epidemiology, this paper distinguishes three types of strategies: Massive Simulation Models (MSMs), Abstract Simulation Models (ASMs) and Macro Equation Models (MEMs). By analyzing some of the main smallpox epidemic models proposed in the last twenty years, it discusses how to justify strategy choice with reference to the core characteristics of these respective strategies. First, I argue that MEMs often

1 Dieser Aufsatz ist eine übersetzte und revidierte Fassung von Grüne-Yanoff (2018).

https://doi.org/10.1515/9783110600261-015 
suffice for policy purposes, and need to be replaced only if they are insufficiently robust. Determining the robustness of MEMs, however, only requires the use of ASMs, not MSMs. Second, I argue that although MSMs have larger potentials than ASMs in various dimensions, they are also more likely to fail - and that in many cases, this probability of failing outweighs their higher potential. In particular, these dimensions include the representation of the relevant target, the accurate measurement of the relevant parameters, the number of parameters included, the number of mechanisms modeled simultaneously, and the ways of dealing with structural uncertainty. While this in no way excludes the prospect that some MSMs provide good justifications for policy decisions, my arguments caution against a general preference for MSMs over ASMs for policy decision purposes in general and vaccination problems in particular.

Epidemieprävention und -kontrolle sind wichtige Funktion der Gesundheitsbehörden. So haben z. B. die Centers for Disease Control and Prevention (CDC) Notfallpläne für Ebola-Epidemien, verschiedene Arten von Grippe, Pocken und viele andere Krankheiten erstellt. Der CDC-Reaktionsplan für Pocken schreibt bspw. eine Impfstrategie vor, die Kontakte einer infizierten Person identifiziert und impft. ${ }^{2}$ Die Annahme dieser Strategie war nicht unumstritten, da einige Autoren die Wirksamkeit des Plans in Frage stellten (z.B. [1]) und stattdessen die freiwillige Massenimpfung der Bevölkerung beim ersten Anzeichen einer Infektion empfahlen.

Entscheidungsträger wie die CDC wollen ihre Entscheidungen auf der Grundlage der besten verfügbaren wissenschaftlichen Erkenntnisse treffen. Jedoch sind Pocken in modernen städtischen Gesellschaften niemals epidemisch aufgetreten, und daher gibt es keine empirischen Belege für die unterschiedliche Wirksamkeit dieser und anderer Impfstrategien, z. B. bei einem möglichen Ausbruch in den USA. Anstelle dessen haben politische Entscheidungsträger und ihre Berater hier auf die Simulierung von Pockenepidemien zurückgegriffen und diese Modelle verwendet, um die Wirksamkeit verschiedener Impfstrategien zu untersuchen.

In diesem Aufsatz untersuche ich, wie Forscher die Modelle wählen, auf deren Basis Impfentscheidungen getroffen werden. Insbesondere werde ich drei Arten von Modellen unterscheiden: Macro-Equation Models (MEMs), Massive Simulation Models (MSMs) und Abstract Simulation Models (ASMs). MEMs kategorisieren die Population in unterschiedliche Gesundheitszustände und modellieren Krankheitsdynamiken zwischen diesen Gruppen. MSMs und ASMs sind im Gegensatz dazu Mikro-Simulationen, welche Infektionen als Interaktion von Individuen modellieren. Jüngste technologische Fortschritte haben die Menge an Details, die bei der Berechnung eines Modells verarbeitet werden können, rasch erweitert. Mikromodelle, deren Details

2 https://www.cdc.gov/smallpox/bioterrorism-response-planning/public-health/vaccination-strategies.html, zuletzt eingesehen am 6.10.2017. 
ausschließlich durch die aktuellen Rechenkapazitäten eingeschränkt sind, nenne ich Massive Simulation Models (MSMs). Modelle, deren Details auch durch andere Überlegungen (Einfachheit, Transparenz usw.) eingeschränkt sind, nenne ich Abstract Simulation Models (ASMs).

Meine Frage ist methodologisch: Mich interessiert, wie eine Modellwahl gerechtfertigt wird. Was rechtfertigt einen Modellierer, bspw. ein MSM einem ASM, oder ein ASM einem MEM vorzuziehen? Antworten auf solche Begründungsfragen hängen offensichtlich vom Zweck ab, für den das Modell verwendet wird. PockenepidemieModellierung erweist sich hier als vortreffliches Fallbeispiel, weil hier alle Modelle zum gleichen Zweck eingesetzt werden - nämlich als Evidenz für die rationale Wahl der Impfstrategie.

Dem ersten Anschein nach mag es scheinen, dass Entscheider MSMs über ASMs oder MEMs bevorzugen sollten, weil MSMs mehr Details enthalten, das reale System besser approximieren können, Komplexität und Heterogenität in der Bevölkerung besser darstellen können und dem Entscheider als holistisches Testbett für mögliche Maßnahmen dienen kann. MEMs und ASMs bieten aufgrund ihrer zusätzlichen Einschränkungen nicht das gleiche Potenzial wie MSM in diesen Aspekten - und mögen daher häufig als mindergeeignet für Politik- und Verwaltungsentscheidungen angesehen werden.

Gegen diese prima facie Intuition wende ich mich in diesem Aufsatz. Entgegen des ersten Anscheins argumentiere ich, dass ASMs oder MEMs für Politik- und Verwaltungsentscheidungen den MSMs häufig vorzuziehen sind. Ich entwickle mein Argument in zwei Schritten. Erstens zeige ich, dass Entscheidungsträger für ihre Zwecke die spezifischen Eigenschaften von MSMs nicht benötigen, wenn MEMs oder AEMs verfügbar sind. Zweitens führe ich eine Reihe von Gründen an, warum MSMs selten ihr Potenzial ausschöpfen. Während MSMs möglicherweise bessere Modelle sein könnten, laufen sie auch mehr Gefahr, gravierende Fehler mit einzuschließen, und sind daher oft nicht besser. Zusammen warnen diese Argumente davor, sich für Politik- und Verwaltungsentscheidungen auf MSM zu verlassen und betonen gleichzeitig die Vorteile, die MEMs und AEMs bieten.

Mein Aufsatz ist wie folgt strukturiert: Unter Abschnitt 1 wird zwischen MEMs, MSMs und ASMs unterscheiden und dieser Unterschied mit Fällen aus der Pockenimpfungsliteratur illustriert. Abschnitt 2 argumentiert, warum MSMs typischerweise nicht benötigt werden, wenn MEMs oder AEMs verfügbar sind. Abschnitt 3 behandelt die speziellen Probleme von MSMs, aufgrund derer ASMs und MEMs häufig vorzuziehen sind.

\section{Drei Typen von Modellen}

Impfung ist eine der wirksamsten Methoden zur Bekämpfung von Epidemien. Viele Impfstoffe bieten jedoch keinen Langzeitschutz oder haben schwerwiegende Nebenwirkungen, sodass eine vorbeugende Impfung (z. B. für alle Kinder im Alter von fünf 
Jahren) nicht möglich ist. Stattdessen sollten diese Impfstoffe nur angewendet werden, wenn das Risiko einer Epidemie ausreichend hoch ist. Der Entscheidungsträger muss dann eine folgenschwere Entscheidung treffen: Wie werden Impfungen in einer großen Population durchgeführt, wenn eine Epidemie unmittelbar bevorsteht oder bereits ausgebrochen ist? Die relevantesten Alternativen hier sind eine Ringimpfung (TV), bei der die potenziellen jüngsten Kontakte einer infizierten Person verfolgt und geimpft werden; eine begrenzte Impfung (LV), bei der eine zufällige Teilmenge der Bevölkerung geimpft wird; oder eine Massenimpfung (MV), bei der die gesamte Bevölkerung geimpft wird. Die Wahl ist nicht trivial: MV ist eher in der Lage, die Ausbreitung der Krankheit zu stoppen, aber ist teurer und trägt Impfrisiken für eine große Bevölkerung. Die Wirksamkeit von LV und TV dagegen sind weniger sicher, aber sie sind weniger kostenintensiv und setzten eine geringere Anzahl von Menschen dem Impfrisiko aus. Die Evaluierung der LV-Impfstrategie im Vereinigten Königreich während der Grippepandemie 2009 [2] gibt einen guten Einblick in die Komplexität dieser Diskussion.

Da in modernen städtischen Gesellschaften Pocken niemals epidemisch aufgetreten sind, gibt es keine direkten empirischen Belege für die unterschiedliche Wirksamkeit verschiedener Impfstrategien. Stattdessen haben die politischen Entscheidungsträger und ihre Berater auf die Simulierung von Pockenepidemien zurückgegriffen und diese Modelle verwendet, um die Wirksamkeit verschiedener Impfstrategien zu untersuchen. Epidemiologen stehen heute eine Vielfalt von Modellen zur Verfügung, auf deren Basis sie Entscheidungen treffen können (für einen Überblick siehe [3]). Um die Diskussion zu vereinfachen, teile ich diese Modelle in drei Gruppen ein: MacroEquation Models (MEMs), Massive Simulation Models (MSMs) und Abstract Simulation Models (ASMs). Obwohl epidemiologische Modelle natürlich detaillierter (oder anders) unterschieden werden können, reicht diese einfache dreigliedrige Unterscheidung für das vorliegende Argument aus. Ich werde diese Kategorien nun der Reihe nach beschreiben, mit einem Beispiel illustrieren und ihre wichtigsten Unterscheidungsmerkmale analysieren.

MEMs abstrahieren die Population in Gruppen mit unterschiedlichem Gesundheitszustand und modellieren Krankheitsdynamiken zwischen diesen Gruppen. Z. B. simulieren Kaplan et al. [4] eine Epidemie in einer Bevölkerung von 10 Millionen, ausgehen von 1.000 Anfangsinfektionen. Dabei wird angenommen, dass die Bevölkerung sich homogen mischt $-\mathrm{d}$. h. alle haben die gleiche Chance, miteinander zu interagieren. Die Basisreproduktionszahl $R_{0}$ wird für die gesamte Simulation als einheitlich angenommen. $R_{0}=3$ wird aus historischen Daten abgeleitet. Ein infiziertes Individuum durchläuft vier Phasen. Nur in der ersten ist der Impfstoff wirksam; nur in der dritten und vierten ist das Individuum ansteckend; in der vierten zeigt sie jedoch Symptome und wird automatisch isoliert. Zusätzlich wird die Verabreichung von Impfungen unter logistischen Bedingungen modelliert: MV der gesamten Bevölkerung wird in zehn Tagen erreicht. Das Aufspüren und Impfen einer infizierten Person in Ringimpfungen erfordert jedoch viermal so viel Aufwand wie eine einfache Impfung. 
Kaplan et al. [4] sind somit ein Beispiel für ein MEM. Die $R_{0}$ ist ein Makroparameter, das die Bevölkerung als Ganzes charakterisiert. Impfeffekte werden direkt an diesem Populationsparameter modelliert, und die Hauptfrage ist, ob die Verabreichung von Impfstoffen die zufällige Verteilung in der Population übertreffen kann. Es überrascht daher vielleicht nicht, dass die Ergebnisse MV viel besser erscheinen lassen als TV. Am fünften Tag nach der ersten Infektion eingeleitet, führt MV zu 560 Todesfällen, während TV zu 110.000 Todesfällen führt. Außerdem ist TV gegenüber der Anzahl der anfänglichen Infektionen sowie gegenüber Änderungen von $R_{0} \mathrm{emp}$ findlicher als MV, was das Urteil zugunsten von MV weiter unterstützt. Die benötigte Zeit für die Identifizierung und Impfung der Exponierten ist einfach zu lang.

Kaplan et al. [4] steht in der Tradition der sog. compartmental models in der Epidemiologie, in der Tradition von Kermack und McKendrick [5]. Zwischen diesen Modellen bestehen zahlreiche Variationen, sowohl hinsichtlich ihrer strukturellen Merkmale als auch ihrer Parametrisierung. ${ }^{3}$ Alle diese Modelle stellen Epidemien als Größenänderung von Bevölkerungsuntergruppen dar (z. B. die susceptible, infected und recovered Gruppen), wobei die Veränderung durch globale Parameter (z. B. $R_{0}$ ) bestimmt wird. Solche globalen Parameter sind Attribute der gesamten Bevölkerung, nicht der Individuen in der Bevölkerung. Aus diesem Grund nenne ich diese Modelle Macro-Equation-Models (MEMs).

MEMs wurden kürzlich für ihre Makroperspektive kritisiert. Z. B. wurde das Kaplan et al. Modell [4] für seine Homogenitätsannahme kritisiert ([6]). Kritiker argumentieren, dass für Pockeninfektion enger Kontakt zwischen infizierten und gesunden Individuen erforderlich ist. In großen Bevölkerungen ist es höchst unplausibel, dass ein infiziertes Individuum mit gleicher Wahrscheinlichkeit Kontakt mit jedem beliebigen Nicht-Infizierten hat. Anstelle dessen scheint es plausibel, dass die Art, wie sich die infizierten Individuen durch die Population bewegen - d. h. mit wem und mit wie vielen gesunden Individuen sie Kontakt haben - Einfluss hat auf den Effekt verschiedener Impfstrategien. Kritiker behaupten daher, dass MEMs keine adäquaten Modelle für Impfstrategie-Entscheidungen seien. Stattdessen schlagen sie Mikrosimulationen für diesen Zweck vor.

Mikrosimulationen (auch bekannt als agent-based models) modellieren die Interaktion von Individuen, einschließlich Individuum-zu-Individuum-Infektionen, um Makrophänomene wie eine Epidemie zu erfassen. Sie können aufgrund ihres individuell ausgerichteten Modellierungsansatzes die oben genannten Homogenitätsannahmen vermeiden. Aus dem gleichen Grund sind Mikrosimulationen rechentechnisch aufwendig. Fortschritte in der Rechnertechnologie haben jedoch in den letzten

3 Beispiele hierfür sind die Wahl zwischen SIR- (susceptible-infected-recovered) und SIS- (susceptible-infected-susceptible) Modellen sowie zwischen verschiedenen Verfeinerungen dieser (z. B. SEIR, MSEIR oder SEIS, vgl. [3]). Das Kaplan et al. [4] Modell gehört zur SEIR-Gruppe, da es zusätzlich zum Standard-SIR-Modell ein „exposed“ Kompartiment enthält. 
Jahren die berechenbare Datenmenge drastisch erhöht. Mikromodelle, deren Details ausschließlich durch die aktuellen Rechenkapazitäten beschränkt sind, nenne ich Massive Simulation Models (MSMs). Modelle, deren Details auch durch andere Überlegungen (Einfachheit, Transparenz usw.) beschränkt werden, nenne ich Abstract Simulation Models (ASMs). Während die genaue Abgrenzung dieser beiden Kategorien notwendigerweise in einer Grauzone liegt, werden die zwei folgenden Beispiele veranschaulichen, wie unterschiedlich Modelle dieser Kategorien sein können.

Mein Beispiel eines ASM: Burke et al. [7] simuliert eine einzige Initialinfektion auf ein städtisches Netzwerk von 6.000 oder 50.000 Menschen. Die Netzwerke bestehen entweder aus einer einfachen Stadt, einem Ring aus sechs Städten oder einer „Narbe“ mit vier „Speichen“. Jede Stadt besteht aus Haushalten mit bis zu sieben Personen, einem Arbeitsplatz und einer Schule. Alle Städte teilen sich ein Krankenhaus. Jede Stadt wird als Gitter dargestellt, sodass jede Zelle im Gitter acht Nachbarn hat. Individuen unterscheiden sich nach Typ (Kind, Gesundheitspersonal [5\% der erwachsenen Bevölkerung], Pendler [10\%] und Nichtpendler [90\%]), nach Familienidentität und Infektionsstatus. An jedem „Tag“ besuchen die Individuen Gitterpositionen entsprechend ihres Typs und kehren dann nach Hause zurück. Am ersten „Tag“ der Simulation wird die Position in Schulen und am Arbeitsplatz zufällig zugewiesen, danach erinnern sich die Individuen an ihre Positionen. Während des „Tages“ interagieren Individuen mit allen ihren unmittelbaren Nachbarn: 10 Mal zu Hause, 7 Mal auf der Arbeit und 15 Mal im Krankenhaus. Die Annahme homogene Mischung wird in diesem Modell also durch eine strukturierte Interaktion von Individuen in dynamischer Nachbarschaften ersetzt.

Die Krankheitsübertragung erfolgt mit einer bestimmten Rate bei Interaktionen der Individuen. Übertragungsraten hängen vom Stadium ab, in dem sich die infektiöse Person befindet, von der Art der Krankheit, und davon, ob natürliche Immunität vorliegt.

Burke et al. bewerteten TV als erste Impfstrategie und LVs unterschiedlichen Grades als Zusatzmaßnahmen. Die Ergebnisse aller drei Netzwerke zeigen eine wesentliche Übereinstimmung. Im Gegensatz zu einem No Response-Szenario reichte TV in Kombination mit einer Krankenhausisolierung aus, um die Epidemie auf weniger als 48 Fälle und eine mittlere Dauer von weniger als 77 Tagen zu begrenzen. Post-Release-LV von $80 \%$ der Gesamtbevölkerung reduzierte den Mittelwert der infizierten Personen auf 33 und verkürzte die mittlere Dauer auf weniger als 60 Tage.

Mein Beispiel eines MSM: Eubank et al. [8] simuliert eine Initialinfektion von 1.000 in der Bevölkerung von Portland/Oregon, von 1,5 Millionen. Portland ist durch ungefähr 181.000 Orte vertreten, jeweils mit einer spezifischen Aktivität, wie Arbeit, Einkaufen oder Schule. Jedes Individuum ist durch eine Liste der Eintritts- und Austrittszeiten für alle Orte gekennzeichnet, die die Person während des Tages besucht hat. Diese riesige Datenbank wurde durch das Verkehrssimulationswerkzeug TRANSIMS entwickelt, das wiederum auf US-Volkszählungsdaten basiert. 
Die Pocken werden durch das Parameter disease load (analog zu einem viralen Titer) modelliert. Individuen haben individuelle Schwellenwerte, oberhalb derer ihre Belastung zu Infektion, Symptomen, Übertragung und Tod führt. Stündlich werfen infektiöse Individuen einen festen Bruchteil ihrer Belastung auf die lokale Umgebung ab. Standorte werden somit mit einer Belastung kontaminiert, die gleichmäßig auf die Anwesenden verteilt wird. Streu- und Absorptionsanteile unterscheiden sich individuell. Infizierte ziehen sich 24 Stunden nach ihrer Ansteckung in ihre Häuser zurück.

Das Eubank et al. [8] Modell ergibt ungefähr ähnliche Ergebnisse für alle Impfstrategien. MV mit einer Verzögerung von 4 Tagen führt zu 0,39 Todesfällen pro anfänglich infizierter Person; TV mit der gleichen Verzögerung zu 0,54 Todesfällen. Unterschiede in der Verzögerungslänge erweist sich in diesem Modell als der wichtigste Faktor bei der Begrenzung von Todesfällen, mit ähnlichen Resultaten für TV und MV.

Beide Modelle geben mehr oder weniger konkrete Entscheidungshilfen. Um nur zwei Beispiele zu nennen: "Contact tracing and vaccination of household, workplace and school contacts, along with effective isolation of diagnosed cases, can control epidemics of smallpox" ([7]:1148); und “Outbreaks can be contained by a strategy of targeted vaccination combined with early detection without resorting to mass vaccination of a population" ([8]:180).

Was sind die Hauptunterschiede zwischen MSMs und ASMs? MSMs unterscheiden sich von ASMs auf den ersten Blick durch ihre viel höhere Detaildichte, insbesondere in der Anzahl der Variablen und Parameter, und der Anzahl der Beziehungen zwischen ihnen. Das Eubank et al. [8] Modell umfasst z. B. ungefähr 1,6 Millionen Knoten mit maximal 1,5 Millionen Kanten, die sich 24 Mal am Tag ändern könnten, während das Burke et al. [7] Modell nur etwa 7.900 Knoten mit maximal 6.000 Kanten umfasst, sie sich maximal $17 \mathrm{Mal}$ pro Tag ändern können.

Basierend auf diesem Reichtum in realistischen Details, wird manchmal behauptet, dass MSMs ein sehr genaues Bild des realen Systems bieten:

Such models allow for the creating of a kind of virtual universe, in which many players can act in complex - and realistic - ways. ([9]:686) ${ }^{4}$

So verstanden, werden MSMs typischerweise als direkte Darstellung realer Systeme gesehen: ihre Struktur ermöglicht eine Abbildung des Modells auf das Zielsystem, ohne Rückgriff auf vermittelnde Modelle zu nehmen. Das Eubank et al. [8] Modell

\footnotetext{
4 Dieses und die folgenden zwei Zitate stammen von Ökonomen, die über ökonomische MSMs schreiben, nicht speziell über die epidemiologischen MSMs, die ich hier diskutiert habe. Debatten in der Ökonomie über die Makro-Mikro-Kluft, über die Genauigkeit und Detaildichte der Modellierung gehen jedoch eng mit den hier angesprochenen Fragen einher. Ich zitiere diese Autoren, um die Motivation für die Wahl von MSM allgemein zu illustrieren, was mutatis mutandis auch auf den betrachteten Fall zutrifft.
} 
wird z. B. als direkte Darstellung der Stadt Portland vorgestellt. ASMs können dagegen kaum behaupten, ein reales System direkt darzustellen - ihr Detaillierungsgrad ist nicht ausreichend. Im besten Fall sind sie in der Lage, stilisierte Tatsachen oder Abstraktionen eines Systems darzustellen, die durch Abstraktion oder Idealisierung aus dem realen System entstanden sind. Burke et al. z. B. erklären explizit, eine „künstliche Stadt“ zu repräsentieren, die einige Eigenschaften mit realen Städten teilt, aber ansonsten anders ist ([7]:1442).

Dass MSMs reale Systeme direkt repräsentieren, wird durch die Praxis unterstützt, das Modell direkt an reale Daten anzupassen oder zu kalibrieren. Das Eubank et al. [8] Modell basiert bspw. auf der Spezifikation von Kanten und deren stündlichen Änderung auf Volkszählungs- und Stichprobendaten über Portland/Oregon. Das Burke et al. [7] Modell dagegen stipuliert bestimmte Kantenänderungen und interpretiert diese Aktivitäten als „zu Hause“, „Weg zur Arbeit“, „im Krankenhaus sein“ usw. Diese Interpretation basiert nicht auf Daten von tatsächlichen Zielsystemen, sondern auf Plausibilitätsannahmen.

Sowohl MSMs und ASMs repräsentieren typischerweise Prozesse ihrer jeweiligen Zielsysteme. Aber auch hier gibt es einen wichtigen Unterschied. MSMs repräsentieren typischerweise eine Vielzahl von parallelen Prozessen oder Mechanismen, während ASMs typischerweise nur eine oder eine kleine Anzahl solcher Prozesse darstellen. Nehmen wir z. B. die folgende Behauptung über die Vorteile von agentenbasierten Modellen in der Ökonomie:

\section{A thorough attempt to understand the whole economy through agent-based modeling will require integrating models of financial interactions with those of industrial production, real estate, government spending, taxes, business investment, and with consumer behavior. ([9]:686)}

Vermutlich werden viele dieser Komponenten durch unterschiedliche Mechanismen operieren. Ein einziges MSM, das alle diese Komponenten repräsentiert, muss viele verschiedene Prozesse gleichzeitig betreiben, um ein Modellergebnis zu erzeugen. Das Eubank et al. [8] Modell unterscheidet bspw. mehrere Aktivitäten an jedem Standort, von denen jeder unterschiedliche Kontaktraten ergibt; es beinhaltet auch die Auswirkungen demographischer Faktoren (insbesondere des Alters) auf die Mischung; es unterscheidet verschiedene Formen von Pocken; und es versucht zumindest einige rudimentäre Auswirkungen der Infektion auf individuelles Verhalten zu integrieren. Das Burke et al. [7] Modell dagegen umfasst eine geringere Anzahl von Standorten und unterscheiden nicht zwischen Aktivitäten, Demographie oder Verhaltenseffekten. Daher enthalten MSM typischerweise viel mehr gleichzeitige Mechanismen als ASMs.

MSMs und ASMs unterscheiden sich außerdem in ihrer typischen Interpretation und Anwendung. MSMs werden aufgrund ihrer größeren Detaildichte häufig als „realistische“ Repräsentationen interpretiert. „Realistisch“ ist ein subjektiver psycho- 
logischer Effekt, der sich aus einem Eindruck von Vertrautheit ergeben mag und zu mehr Vertrauen in das Modell und seine Schlussfolgerungen führen könne:

Decision makers might be more willing to trust findings based on rather detailed simulation models where they see a lot of economic structure they are familiar with than in general insights obtained in rather abstract mathematical models. ([10]:354)

Im Eubank et al. [8] Modell kann der Benutzer z. B. die Entwicklung der Epidemie auf einer Karte von Portland/Oregon verfolgen. Die Karte bietet eine hochauflösende Darstellung der Wohndichte und mit fortschreitender Epidemie den Anteil der Bevölkerung, die an einer Position infiziert ist, sowie die Gesamtanzahl der Todesfälle, Impfungen und Quarantänen. Der Benutzer erhält so einen „realistischen“ Eindruck von einer möglichen Epidemieentwicklung in der Stadt. ASMs bietet keine so reiche Sammlung bekannter Details und werden daher in der Regel nicht als „realistisch“ interpretiert.

Dies führt auch zu einem wichtigen Unterschied in der Verwendung dieser Modelltypen für Politik- und Verwaltungsentscheidungen. MSMs werden häufig als Teil eines „holistischen Ansatzes“ verwendet: Ein „Modell der gesamten Wirtschaft“ wird als „virtuelles Universum“ verwendet ([9]:686), um die Auswirkungen vorgeschlagener Interventionen zu bewerten im Zielsystem. Das heißt, Interventionen werden im Modell simuliert und Modellresultate werden als Prognosen der Ergebnisse solcher Interventionen im realen System interpretiert. Während Eubank et al. [8] eine solche Verwendung ihres Modells nicht ausdrücklich befürworten, ist es zumindest damit vereinbar. ASMs können nicht auf diese Weise verwendet werden, da es keine (scheinbare) Darstellung des gesamten Systems bietet. Die holistische Synthese, die das MSM verspricht, muss vom ASM-Nutzer auf andere Weise durchgeführt werden, z. B. durch Expertenrunden.

Natürlich haben MSMs und ASMs trotz der Unterschiede wichtige Ähnlichkeiten. Beide zielen darauf ab, nichtlineares und komplexes Verhalten darzustellen, wenn auch auf verschiedenen Abstraktions- und Idealisierungsebenen. Dadurch unterscheiden sie sich von MEMs. Darüber hinaus abstrahieren und idealisieren beide Modelltypen, aber in unterschiedlichem Maße und aus verschiedenen Gründen. MSMs abstrahieren und idealisieren aus Gründen der Lenkbarkeit und der Berechenbarkeit: Sie sind hauptsächlich durch die derzeitigen Rechenkapazitäten eingeschränkt. ASMs im Gegensatz dazu werden auch durch andere Überlegungen (z. B. der Einfachheit, der Transparenz usw.) bestimmt, sodass ihre Berechenbarkeit selten zu einer relevanten Einschränkung für sie wird.

Schließlich ist die Unterscheidung zwischen MSM und ASM selbst eine Vereinfachung. Viele tatsächliche Simulationsmodelle weisen einige Eigenschaften des einen Typs und einige des anderen auf und fallen somit nicht eindeutig in eine der beiden Kategorien. Dies ist jedoch kein Problem für meine Argumentation; meine Diskussion 
im Abschnitt 3 bespricht jede dieser Eigenschaften separat, sodass relevante Schlüsse für solche Hybridtypen gezogen werden können.

\section{MSMs nicht notwendig für politische Entscheidungen}

Epidemiologen stehen vor einer methodologischen Entscheidung: Sie müssen aus einer großen Auswahl möglicher Modelle das Modell auswählen, das sie verwenden wollen, und sie müssen begründen, warum dies die beste Wahl für ihre Zwecke ist. In der Praxis wird dieses Menü natürlich aus einer unübersichtlichen Menge von Möglichkeiten bestehen. Aber für meine Argumentation reicht es aus, dieses methodologische Problem als eine Wahl zwischen drei abstrakten Typen von Modellen - MEMs, ASMs und MSMs - zu diskutieren. Mein Ziel ist es, die Gründe für die Wahl einer Option gegenüber einer anderen darzustellen und zu analysieren, wenn der Zweck dieser Modellierung die Unterstützung von Politik- und Verwaltungsentscheidungen ist.

In diesem Abschnitt zeige ich, dass die Eigenschaften, welche MSMs gegenüber MEMs und AEMs auszeichnen, für die Zwecke von politischen und Verwaltungsentscheidern nicht vonnöten sind. Fürsprecher von MSMs mögen zwar argumentieren, dass MSMs im Rahmen eines „ganzheitlichen Ansatz“ verwendet werden können: das Model wird als exakte Kopie des realen Systems interpretiert, sodass jeder im Modell simulierte Effekt einer Entscheidung als verlässliche Vorhersage solcher Eingriffe im realen System benutzt werden kann. Während eine solche Sichtweise offensichtlich eine große Anziehungskraft auf Entscheidungsträger ausübt, ist sie nicht notwendig, um Entscheidungen rational zu legitimieren.

Tatsächlich können die meisten Formen der systematischen Kontrolle ohne solche ganzheitlichen Kopien erfolgreich durchgeführt werden. Als Beispiel sei der Kapitän genannt, der ein Boot durch raue See steuert. Um sein Schiff auf sicherem Kurs $\mathrm{zu}$ halten, muss er nicht alle Faktoren voraussagen, die den Kurs des Bootes beeinflussen, noch muss er das Zusammenspiel dieser Faktoren mit dem Lenkmechanismus vorhersagen. Vielmehr genügt es, dass er den Lenkmechanismus kennt und anzuwenden weiß, um den Bootskurs beeinflussen zu können. Andere Faktoren (z. B. Wind, Strömung, Hindernisse) beeinflussen natürlich auch die Bootsleistung. Es genügt jedoch, dass der Kapitän richtig beobachtet, wie sich die gegenwärtigen Faktoren auf den Kurs auswirken, und dass er das Steuer entsprechend korrigiert, um die Kontrolle über das Boot zu behalten.

Ähnlich ist es mit Maßnahmen zur Steuerung einer Epidemie. Solange der Entscheider zuverlässige Mechanismen kennt, durch die er den Verlauf der Epidemie beeinflussen kann, und anzuwenden weiß, muss er weder in der Lage sein, die Entwicklung des gesamten Systems vorherzusagen, noch die Wechselwirkung von anderen Teilen des Systems mit seinen Maßnahmen genau vorherzusagen. Stattdessen ist es legitim, dass die Anwendung dieses Wissens von den beobachteten Bedingungen des Zustands der Epidemie und ihrer Entwicklung abhängt, wie es hier ausgedrückt wird: 
Would an attack be small and controllable through traced vaccination or large enough to require mass vaccination? Would an attack be overt, in which case it could prove possible to respond immediately in a highly targeted fashion and obtain much better results, or covert and detected only from symptomatic cases as assumed in this article? ([11]:269)

Kaplan kann diese genauen Fragen stellen, weil er in seinen Modellen einen vermeintlich zuverlässigen Mechanismus zur Kontrolle „kleiner“ Epidemien isoliert, einen anderen für solche, die „large enough“ sind, einen für „offene“ Infektionen und einen für „verdeckte“. Ein solches Wissen ermöglicht es den Entscheidungsträgern auf die Bedingungen einer tatsächlichen Epidemie zu reagieren, indem sie den geeigneten Mechanismus wählen, der es ihnen ermöglicht, das System in die gewünschte Richtung zu beeinflussen - ohne jedoch notwendigerweise die Entwicklung des gesamten Systems vorherzusagen zu können.

Das Konzept der isolierenden Modelle ist in der Literatur ausführlich analysiert (z. B. [12],[13]). Ein kausaler Zusammenhang zwischen einer Intervention und einem Effekt wird isoliert, indem die Auswirkungen anderer störender Faktoren aus dem Modell ausgeschlossen werden. Entweder werden diese Faktoren aus der Darstellung vollständig weggelassen oder sie werden idealisiert (d. h. ihren Einflussparametern werden bekanntermaßen nicht-akkurate Werte zugeordnet, häufig null oder unendlich).

Allerdings muss man den Isolationsprozess, der eine Idealisierung beinhalten kann, vom Produkt der Isolation unterscheiden. Um einen Faktor $F$ von anderen Faktoren $G_{1}, \ldots, G_{\mathrm{n}} \mathrm{zu}$ isolieren, können die Einflüsse der $G_{\mathrm{i}}$ idealisiert werden, nicht aber den Einfluss des Faktors $F$ selbst. Auf diese Weise trifft das Modell zwar falsche Behauptungen über die $G_{\mathrm{i}}$; aber das Ziel des theoretischen Prozesses - die Isolierung von $\mathrm{F}$ - bleibt intakt. Idealisierung ist also ein Verfahren, das auf Entitäten angewandt wird, von denen man isoliert, aber nicht auf Entitäten, die man isolieren will ([13]:328); und Idealisierung wird als Hilfstechnik zur Erzeugung von Isolation verwendet, ist aber kein Teil der Isolation selbst ([13]:325). So wird das Isolationsprodukt - der isolierte Faktor - niemals idealisiert. In Kaplans MEM-Modell wird bspw. die Entwicklung der Epidemie dadurch isoliert, dass demografische Entwicklungen (VitalDynamics) überhaupt nicht berücksichtigt werden. Während eine solche Annahme offensichtlich falsch ist, kann das Modell die isolierte Wirkung der Intervention auf die Krankheit weiterhin korrekt darstellen.

Diese Analyse hilft uns zu verstehen, wann es MEMs gelingt, Informationen über zuverlässige Mechanismen zu liefern und wann dies nicht der Fall ist. Kaplans Modell enthält z. B. viele idealisierende Annahmen, einschließlich "all random tracing is of susceptible", oder "all people leave the untraced compartment via tracing, not disease symptoms" ([14]:46). Diese Idealisierungen sind eindeutig Annahmen über den isolierten Mechanismus selbst - d.h. über die Relation zwischen Intervention und Epidemie. Daher untergraben sie die oben genannte klare Trennung zwischen isolierten und idealisierten Entitäten ([15],[16]). 
Solche Idealisierungen der Mechanismen selbst müssen jedoch nicht notwendigerweise die Relevanz eines Modells gefährden. Stattdessen könnte ein Modellierer zeigen, dass eine solche idealisierende Annahme tatsächlich harmlos ist. Durch Variieren der fraglichen Annahme und der Beobachtung, dass sich das Modellergebnis unter solchen Variationen nicht ändert, zeigt ein Modellierer, dass das Modell unter der fraglichen Annahme derivativ robust ist. Robustheitsergebnisse erlauben dem Modellierer darauf zu vertrauen, dass die Annahme, obwohl sie einen Teil des Mechanismus idealisiert, das Ergebnis des Modells nicht beeinflusst [17]. Wenn dies der Fall ist, gibt es wenig Grund zu befürchten, dass die Zuverlässigkeit des Modells durch diese Annahme beeinträchtigt wird.

Robustheit spielt eine wichtige Rolle in der Debatte um homogenes Mischen bei der Pockenmodellierung. Wie oben erwähnt, kritisierten Halloran et al. [6] das Kaplan et al. MEM [4] wegen dessen Vernachlässigung der Netzwerkstruktur. Kaplans et al. MEM ergab bessere Resultate durch MV als durch TV. Doch als Halloran et al. die Homogenitätsannahme durch eine strukturierte Interaktion zwischen Individuen ersetzten, ergab MV nicht mehr bessere Resultate als TV. Kaplan und Wein [18] antworteten darauf, dass dieser Unterschied nur durch eine Differenz in den Bevölkerungsgrößen der jeweiligen Simulationen zustande kam. Wenn beide Modelle im gleichen Maßstab verglichen werden, zeigten sie, dass beide Modelle in etwa die gleichen Ergebnisse liefern (nämlich MV effektiver als TV). Halloran und Longini [19] antworteten, dass dies zwar für kleine Populationen und eine geringe Anzahl von Erstinfektionen zutrifft, dies jedoch für größere Populationen und viele anfängliche Infektionen unwahrscheinlich ist. ${ }^{5}$

Diese Debatte veranschaulicht, wie solche Robustheitsanalysen ablaufen. Halloran et al. [6] hatten einige prima facie Gründe zu glauben, dass die Homogenitätsannahme das isolierende Modell für die Entscheidung unzuverlässig machen würde. Sie konstruierten daher ein ASM ohne diese und zeigten, dass dies einen Unterschied für die vorliegende Entscheidung machte. Kaplan und Wein [18] konnten jedoch zeigen, dass es nicht diese Idealisierung war, die einen Unterschied machte, sondern eher der Maßstab. Das MEM war somit robust in Bezug auf die Homogenitätsannahme, zumindest in einem kleinen Maßstab (d.h. kleine Populationen mit geringer Anzahl anfänglicher Infektionen). Es blieb jedoch unklar, ob das MEM auch in größerem Umfang robust sein würde. Halloran und Longini [19] können kein Ergebnis vorweisen, welches das Gegenteil zeigt, geben aber verschiedene Plausibilitätsgründe an, die dagegensprechen.

5 Allerdings modellierten Halloran et al. solche großen Bevölkerungsgrößen nicht, sondern spekulieren nur über solche Situationen: "Many choices are available in scaling up the heterogeneous [ASM] model ... Difference between the two current models [Kaplan's et al. MEM and their ASM] could also become more manifest when scaled up" ([6]: 1503). 
Ohne diese Debatte entscheiden zu wollen, kann man dennoch feststellen, dass die Argumente von beiden Seiten sich auf die Verwendung sowohl von MEMs und ASMs stützen. Halloran et al. [6] brauchten das ASM, um die Idealisierung in Frage zu stellen. Auch Kaplan und Wein [18] benötigten das ASM, um zu zeigen, dass diese DeIdealisierung keinen Unterschied machte. Halloran und Longini [19] würden darüber hinaus ein modifiziertes ASM (aus den ,vielen Möglichkeiten ... zur Vergrößerung“) benötigen, um ihre Behauptung zu stützen, dass das MEM im größeren Maßstab nicht robust ist. Robustheit erfordert einen Modellvergleich über verschiedene Arten von Modellen hinweg - und insbesondere in der Epidemiologie zwischen MEMs und ASMs. Selbst wenn am Ende alle zustimmten, das MEM als das relevante Modell zu akzeptieren, hätten ASMs eine wichtige Beweisfunktion in dieser Annahmeentscheidung gespielt.

Das MSM jedoch wird für diesen Zweck nicht benötigt. Warum nicht? Weil der für Robustheitsfragen relevante Modellvergleich immer nur die De-Idealisierung einer oder einiger weniger Annahmen betrifft. Um dies zu tun braucht man keine massiven Details, mehrere Mechanismen, direkte Darstellung eines realen Ziels oder Kalibrierung mit realen Daten. Zwar musste in dem vorliegen Fall von einer Makro- zu einer Mikrosimulation gewechselt werden. Diese Mikrosimulation konnte jedoch in vielerlei Hinsicht stark vereinfacht sein. ASMs waren dafür ausreichend. Daher benötigt der Entscheider kein MSM für seine Zwecke.

\section{MSMs enthalten eher Fehler als ASMs}

Obwohl MSMs nicht notwendig sind, um zu verlässlichen Belegen für die Politikgestaltung zu gelangen, wäre es natürlich für einen politischen Entscheidungsträger hilfreich, ein Modell zu haben, das den genauen Verlauf einer Epidemie im Voraus einschließlich aller Umwelteinflüsse korrekt vorhersagt und außerdem korrekte Vorhersagen über die Wirkung aller möglichen Interventionen trifft.

Während solch eine Verwendung natürlich wünschenswert wäre, argumentiere ich hier, dass MSM selten die Bedingungen erfüllen, unter denen die Hoffnung auf eine solche Verwendung gerechtfertigt wäre. Speziell zeige ich, dass MSMs zwar größere Potenziale als ASMs in verschiedenen Dimensionen haben, aber auch eher scheitern - und dass diese Wahrscheinlichkeit des Scheiterns in vielen Fällen ihr höheres Potenzial überwiegt. Unter Verwendung der obigen Pockenmodelle diskutiere ich nun die verschiedenen Dimensionen, in denen MSMs im Vergleich zu ASMs versagen könnten und weswegen deshalb letztere vorzuziehen sind. Konzeptionell trenne ich diese Dimensionen, obwohl sie in der Praxis häufig überlappen. 


\subsection{Was ist das Zielobjekt?}

MSMs wie z. B. bei Eubank et al. [8] sind prima facie auf ein bestimmtes Objekt gerichtet: z. B. die Stadt Portland/Oregon. ASMs wie bei Burke et al. [7] dagegen scheinen kein solches Ziel zu haben; sie repräsentieren vielmehr einen abstrahierten Typ, wie „eine Stadt“ oder „ein städtisches Bevölkerungsnetz“. Folglich werden MSMs oft als realistischer als ASMs bewertet, da Modellbenutzer die MSM-Merkmale leichter auf die Eigenschaften eines bestimmten Ziels zurückführen können. Diese Einschätzung der Realitätsnähe wiederum, wie das obige Zitat von Dawid und Fagiolo [10] zeigt, veranlasst Entscheider oft dazu, mehr Vertrauen in die Zuverlässigkeit und Nützlichkeit des fraglichen Modells zu setzen. Aus diesem Grund scheinen sie MSMs häufig ASMs für ihre Zwecke vorzuziehen.

Aber ist dieser Schluss von der Einschätzung der Realitätsnähe auf die Zuverlässigkeit und Nützlichkeit gerechtfertigt? Vermutlich lautet das Argument, dass (i) ein realistisches Modell eine sehr genaue Darstellung des Ziels ist und dass (ii) eine genaue Darstellung des Ziels eine notwendige Bedingung ist, um zuverlässige Informationen über mögliche Eingriffe zu erlangen.

Während ich diese Behauptungen hier nicht einzeln bestreite, argumentiere ich, dass ihre Verbindung kein gültiges Argument darstellt, wenn sich das Zielobjekt zwischen ihnen ändert. Genau das aber passiert in den Pocken-Simulationsstudien. Ziel der Entscheidung ist ein generisches städtisches Umfeld, wie der einleitende Satz von Eubank et al. zeigt:

The dense social-contact networks characteristic of urban areas form a perfect fabric for fast, uncontrolled disease propagation. [...] How can an outbreak be contained before it becomes an epidemic, and what disease surveillance strategies should be implemented? ([8]:180)

Da epidemische Maßnahmen in der Regel in der Verantwortung von nationalen oder internationalen Institutionen liegen, sind die Ziele der Entscheider alle Städte innerhalb des Regierungsbereichs dieser Institution (z. B. alle US-Städte, alle Städte in Industrieländern, alle Städte der Welt usw.). Das Ziel einer solchen Entscheidung ist somit eine abstrakte Einheit: die Netzwerkeigenschaften aller städtischen Gebiete innerhalb des relevanten Bereichs.

Das Ziel der Pocken-MSM dagegen ist kein generisches, sondern ein konkretes Objekt: die Stadt Portland/Oregon. Die Autoren dieses Modells behaupten, dass es sich hierbei nur um eine Instanziierung der Netzwerkcharakteristika handelt. ${ }^{6}$ Indem sie jedoch ein konkretes Ziel auswählen, ermöglichen sie eine mögliche Divergenz zwischen der Bedeutung von „Ziel“ in Schritt (i) und (ii) in dem obigen Argu-

6 "We view the social networks created by TRANSIMS as a single instance of a stochastic process defined in an enormous space of possibilities” ([8] Supplement:3). 
ment. Insbesondere könnte die Beurteilung, dass ihr Modell realistisch ist, nur auf Ähnlichkeiten ihres Modells und der Stadt Portland basieren, die für Ähnlichkeiten zwischen ihrem Modell und den Netzwerkeigenschaften aller städtischen Gebiete innerhalb der relevanten Domäne völlig irrelevant sind. Z. B. könnte die Einbeziehung des Columbia-Flussbettes, der Standorte von Portlands Universitäten sowie des öffentlichen Transportsystems von Portland die realistische Beurteilung des Modells erhöhen. Dies können jedoch Merkmale sein, die für den Weg einer Epidemie durch ein städtisches Netzwerk entweder irrelevant sind oder für urbane Netzwerke in den USA generell nicht repräsentativ sind. In beiden Fällen wäre die Verbindung zwischen Reliabilität, Zuverlässigkeit und Nutzen aufgehoben: Ein MSM mit diesen Merkmalen ist möglicherweise realistischer als ein ASM, während der ASM eine genauere Darstellung der relevanten Netzwerkeigenschaften aller städtischen Gebiete darstellt. In solchen Fällen wäre das ASM ein leistungsfähigeres Entscheidungsinstrument als das MSM.

\subsection{Parameter-Messung}

MSMs unterscheiden sich von ASMs in ihrem viel höheren Detaillierungsgrad, insbesondere der Anzahl der Variablen und Parameter, die sie enthalten und der Anzahl der Beziehungen zwischen diesen. Unter der Annahme, dass beide Modelle das gleiche Ziel haben (sodass das Problem des vorangegangenen Abschnitts nicht auftritt), gibt eine höhere Anzahl von Variablen und Parametern MSMs mehr Potenzial als ASMs, um das Zielsystem genau darzustellen. Prima facie bietet dies MSMs einen Vorteil gegenüber ASMs für Politik- und Verwaltungsentscheidungen.

Dieses Argument geht jedoch davon aus, dass die zusätzlichen Variablen und Parameter, die MSMs einen Vorteil gegenüber ASMs geben, mit ausreichender Genauigkeit gemessen oder geschätzt werden können. Beide Annahmen sind problematisch. Ich werde die Messprobleme in diesem Teilabschnitt und die Schätzprobleme im nächsten Abschnitt besprechen.

Die gemessenen Variablen und Parameter der Pocken-MSM sind diejenigen, deren Wert direkt von einer externen Datenquelle geliefert wird. Z. B. werden Eigenschaften wie Alter, Beruf, Gesundheit und Heimatstandort aus Volkszählungsdaten für alle 1,5 Millionen Individuen in dem Modell erhalten. Die Eigenschaften des städtischen Verkehrsnetzes und der Landbesetzung und -nutzung werden von städtischen Planungsorganisationen erhalten ([8] Supplement:3). Diese Beispiele für eine massive Datenaufnahme scheinen tatsächlich den vergleichenden Detailreichtum von MSMs gegenüber ASMs zu unterstützen.

Eine genauere Lektüre des Artikels und seines ergänzenden Materials zeigt jedoch, dass viele der Parameter und Variablen nicht genau gemessen (oder überhaupt gemessen) werden konnten. Sie werden vielmehr durch Ad-hoc-Annahmen, oder Ver- 
mutungen bestimmt. Ich beschreibe hier drei Beispiele zur Veranschaulichung. Das erste betrifft die krankheitsrelevanten Kontakte von Individuen:

We do not have data for proximity of people, other than that they are in the same (possibly very large) location. [...] It seems as though the dependence on distance is very coarse: one mode of transmission occurs at close ranges ( 6 feet) and another for large ranges. We have developed an ad hoc model that takes advantage of this coarseness. ([8] Supplement:9)

Dieses Ad-hoc-Modell macht einheitliche Annahmen über die Belegungsrate von Standorten innerhalb eines Stadtblocks, die, so die Autoren, „nichts weiter als vernünftige Vermutungen“ sind ([8] Supplement:11). Die Auslastung der Standorte beeinflusst jedoch entscheidend die Anzahl der möglichen Kontakte - und kann daher für die Ausbreitung von Krankheiten relevant sein.

Ein anderes Beispiel betrifft die Parametrisierung des Krankheitsmodells:

There is not yet a consensus model of smallpox. We have designed a model that captures many features on which there is widespread agreement and allow us to vary poorly understood properties through reasonable ranges. ([8]:183)

Was reasonable in diesem Zusammenhang bedeutet und in welchem Umfang es mit den verfügbaren Daten zusammenhängt, bleibt unklar. Zum Schluss noch ein Beispiel zur Parametrisierung der TV-Intervention:

Every simulated day, if contact tracing is in effect, a subset of the people on the list [of people showing symptoms] is chosen for contact tracing. [...] In the experiment reported here, we use the fraction 0.8 and set the absolute threshold at either 10,000 or 1,000. These are probably unrealistic numbers, but they allow us to estimate the best case results of a targeted vaccination strategy. ([8] Supplement:11)

In all diesen Beispielen stellt sich das MSM nicht als detailreich, sondern als detailfordernd heraus: die große Menge an Parameter und Variablen, welche MSMs gegenüber ASMs charakterisieren, wirft die Frage auf, wie diese mit Inhalt gefüllt werden können. Standardmäßig könnte man annehmen, dass sie mit empirischen Daten gefüllt sind. Für diese Beispiele lagen jedoch keine empirischen Daten oder Daten zu geringer Qualität vor. Die Modellierer griffen stattdessen auf Ad-hoc-Annahmen oder beste Vermutungen zurück.

Ich beabsichtige diese Beobachtungen nicht als Kritik an dem bestimmten Pockenmodell oder an MSMs im Allgemeinen. Es erscheint durchaus sinnvoll, einige Parameter des eigenen Modells zu improvisieren. Aber wenn man die Modellwahl diskutiert und insbesondere die Detailauflösung des Modells wählt, sollte man berücksichtigen, wie diese Entscheidung den Improvisationsbedarf beeinflusst. Stellen Sie sich einen Extremfall vor, in dem ein detailarmes Modell mit nur wenigen Parametern, die alle aus qualitativ hochwertigen Daten bestimmt werden können, zu einem 
detailreichen Modell entwickelt werden kann, dessen Parameter nur durch Ad-hocAnnahmen, Bestwerte oder durch Intervallschätzungen bestimmt werden können. Da diese Improvisationen eine große Fehlerwahrscheinlichkeit aufweisen, ist das detailarme Modell wahrscheinlich genauer und daher für Entscheidungszwecke vorzuziehen, als das detailreiche Modell. Mein MSM- vs. ASM-Fall ist viel weniger klar als dieser Extremfall, erstens, weil manche Parameter des ASM auch durch Improvisation bestimmt werden, und zweitens, weil das MSM viele zertifizierte Daten enthält. Dennoch zeigt sich ein ähnliches Kompromissmuster wie im Extremfall und dieser Trade-off könnte in einigen Fällen zu der Schlussfolgerung führen, dass das ASM ein leistungsfähigeres Politikinstrument ist als das MSM.

\subsection{Anzahl der Parameter}

Nehmen Sie an, dass die Messung der Parameter kein Problem darstellt, sodass 3.2 keine Beschränkungen für die Detailtiefe in einem MSM auferlegen würde. In diesem Fall ergibt sich ein anderes Argument gegen eine solche unbegrenzte Zunahme an Details, basierend auf der vergleichbaren Leistung solcher Modelle bei der Parameterschätzung oder -kalibrierung.

Abgesehen von den technischen Details zielen sowohl Schätzungen als auch Kalibrierungen darauf ab, Werte von nicht beobachtbaren Modellparametern zu bestimmen, indem das Modell an beobachtbare Daten angepasst wird. Im Pockenfall werden so viele Parameter der zugrundeliegenden TRANSIMS- und EpiSims-Modelle bestimmt. Um es einfach auszudrücken, das Modell nimmt Volkszählungsdaten, Transportnetzwerkdaten, Landnutzungsdaten usw. als Eingaben auf und gibt als Output Kontaktinzidenz, -dauer und -position zwischen Individuen an. In Übereinstimmung mit dem generative approach in Simulationsstudien [20] werden dann Modellparameter angepasst, um dasjenige Modellergebnis zu erzeugen, das am besten zu den Beobachtungsdaten passt. Sobald eine solche Annäherung erreicht wurde, wird das Modell als validiert betrachtet und es werden kontrafaktische Interventionen eingeführt.

Auf den ersten Blick scheinen MSMs für Schätzungen oder Kalibrierungen besser geeignet zu sein. Wenn das Zielobjekt von hoher Komplexität ist (was im Falle der Impfpolitik zweifellos der Fall ist), ist das Modell umso weniger gut geeignet, auf das Ziel zu passen, je mehr Beschränkungen dem Modell auferlegt werden (z. B. die Art und Anzahl seiner Parameter). Umgekehrt gilt, je weniger Beschränkungen einem Modell auferlegt werden, desto besser kann es auf sein Ziel passen. So scheint es, dass MSMs besser auf ihre Ziele passen als ASMs und daher als das leistungsfähigere Entscheidungsinstrumente erscheinen.

Die obige Intuition, obwohl korrekt, verfehlt ein wichtiges Dilemma, das aus der Modellauswahlliteratur gut bekannt ist. Obwohl Modelle mit mehr freien Parametern ein größeres Potenzial für die Anpassung an das Ziel aufweisen, führt die größere 
Anzahl an freien Parametern in der Praxis oft zu einer geringeren Anpassung als die eines Modells mit weniger Parametern.

Dieses Dilemma wird verständlicher, wenn zwei Schritte im Prozess der Anpassung eines Modells an Daten unterschieden werden. Der erste Schritt besteht darin, die Struktur des Modells, d. h. die Anzahl der Parameter, zu bestimmen. Hier erhöht die Anzahl von Parametern tatsächlich das Potenzial des Modells, das Ziel genau darzustellen.

Der zweite Schritt besteht darin, die Parameter basierend auf einer Stichprobe aus der Population zu kalibrieren oder zu schätzen. Die Erhöhung der Anzahl der Parameter erhöht zwar die Anpassung des Modells an die Stichprobe - aber das ist nicht das ultimative Ziel. Vielmehr soll die Anpassung des Modells an die Population verbessert werden. Wird das Modell zu „nah“ (d. h. durch zu viele Parameter) an die Probe angepasst, werden unvermeidliche zufällige Fehler in der Probe repräsentiert, was zu einer Zunahme der Divergenz zwischen Modell und Ziel führt. Dieses Phänomen ist in der Statistik und der maschinellen Lernliteratur als overfitting bekannt und gilt auch für die Simulationsmodellierung [21].

Die Auswahl der richtigen Anzahl von freien Parametern ist somit das Problem, "finding an appropriate compromise between these two opposing properties, potential and propensity to underperform” ([22]:45). Wie verschiedene Studien gezeigt haben, wird bei einem großen Stichprobenumfang das Hinzufügen weiterer Parameter über einen bestimmten Schwellenwert die Anpassung an das Ziel nicht wesentlich verbessern. Wenn die Stichprobengröße mittel oder klein ist, verringert das Hinzufügen weiterer Parameter sogar die Anpassung an das Ziel [22],[23].

Diese allgemeine Feststellung gilt auch für die Wahl zwischen MSM und ASM. In Abschnitt 3.1 habe ich festgelegt, dass MSMs viel mehr Parameter enthalten als ASMs. Folglich unterliegen MSMs eher der Gefahr von overfitting und sind daher eher anfällig dafür, die unterliegende Population ungenügend abzubilden. Natürlich muss für den Einzelfall entschieden werden, welches Problem überwiegt (insbesondere gilt dies auch für die beiden Pockenmodelle, da eine numerische Untersuchung ihrer jeweiligen Passung den Rahmen dieses Artikels sprengt). Dennoch macht diese generelle Tendenz es unplausibel MSMs allgemein gegenüber ASMs zu bevorzugen.

\subsection{Anzahl der Mechanismen}

Eines der wichtigen Merkmale der hier diskutierten Simulationsmodelle ist, dass sie explizit darauf abzielen Mechanismen darzustellen. Im Fall der Pocken wurden sowohl das MSM als auch das ASM als Verbesserung gegenüber Kaplans et al. Makromodell [11] gesehen, weil sie explizit den Populationsmischungsmechanismus modellierten, anstatt einfach eine homogene Mischung anzunehmen. Dennoch unterscheiden sich MSM und ASM erheblich darin, wie sie solche zusätzlichen Mechanismen einführen. Das Pocken-ASM versucht eine kleine Anzahl einfacher Mechanismen einzuführen, 
während das MSM eine Vielzahl von detailreichen Mechanismen einführt, von denen angenommen wird, dass sie parallel operieren.

Insbesondere unterscheidet das MSM mehrere Aktivitäten an jedem Standort, von denen jede unterschiedliche Kontaktraten ergibt; es beinhaltet auch die Auswirkungen demographischer Faktoren (insbesondere des Alters) auf die Mischung; es unterscheidet verschiedene Formen von Pocken; und es versucht zumindest einige rudimentäre Auswirkungen der Infektion auf das Verhalten der Individuen zu integrieren. Das ASM hingegen umfasst eine geringere Anzahl von Standorten und unterscheidet nicht zwischen Aktivitäten oder demografischen Merkmalen; außerdem repräsentiert es keine Infektionseffekte auf das Verhalten.

Den meisten Beobachtern erscheinen diese zusätzlichen Mechanismen als vorteilhaft. Deswegen scheint es dann auch prima facie plausibel zu sein, das MSM dem ASM vorzuziehen, da ersteres noch mehr Mechanismen und mechanistische Details als letzteres enthält.

Gegen diese Intuition möchte ich ein Argument gegen die vermeintlich höhere Erklärungskraft realistischer Simulationsmodelle anbringen. Dieses Argument wurde u. a. von Lenhard und Winsberg [17] mit einem speziellen Fokus auf Klimamodelle entwickelt. Diese Autoren argumentieren, dass Modelle mit zunehmender Komplexität immer undurchsichtiger werden und dass diese Opazität das Verständnis der Beiträge der Modellkomponenten zum Modellergebnis verhindert oder zumindest reduziert.

Genauer gesagt argumentieren Lenhard und Winsberg, dass mit zunehmender Komplexität die fuzzy modularity eines Modells zunimmt. Je komplexer ein Modell ist, desto mehr Unterkomponenten hat es. Wenn eine Simulation für ein komplexes Modell ausgeführt wird, werden diese Modellkomponenten zusammen und parallel ausgeführt. Aber sie tragen nicht alle unabhängig zum Modellergebnis bei. Vielmehr tauschen die Komponenten im Verlauf einer Simulation häufig Ergebnisse intermediärer Berechnungen untereinander aus, sodass der Beitrag jeder Komponente zum Modellergebnis wiederum von all jenen Komponenten beeinflusst wird, die damit interagieren.

The results of these modules are not first gathered independently and then only after that synthesized. [...] The overall dynamics of one global climate model is the complex result of the interaction of the modules - not the interaction of the results of the modules [...]. Due to interactivity, modularity does not break down a complex system into separately manageable pieces. ([24]:258)

Anders ausgedrückt ist der Effekt der multiplen Mechanismen in einem MSM eher unterbestimmt als in einem ASM: aufgrund der größeren Anzahl von Mechanismen, die in einem MSM enthalten sind, aber auch aufgrund der erhöhten Interaktion - der fuzzy modularity - der Mechanismen im MSM. Offensichtlich gibt es in einem MSM wie bei Eubanks et al. [8] mehr fuzzy modularity als in einem ASM wie bei Burke et al. [7]. In erster Linie ist dies ein Problem für die Erklärungskraft von MSMs. Obwohl MSMs möglicherweise das explanandum ziemlich exakt generieren, ist es wegen der 
höheren Unterbestimmtheit in MSMs schwieriger als in ASMs, darauf zu schließen, welche Ursachen durch welche Mechanismen das Ergebnis erzeugt haben. Wenn aber Verständnis darin besteht, die Mechanismen zu identifizieren, die das explanandum hervorgebracht haben, dann untergräbt die fuzzy modularity eines Modells die Verbesserung unseres Verständnisses.

Diese Sorge gilt auch für die Nutzung von MSMs als Entscheidungsinstrument. Das Modell und die Simulation sollen Entscheidern dabei helfen Interventionen zu identifizieren, die in den relevanten Kontexten die gewünschten Ergebnisse zuverlässig produzieren. Wenn wir eine solche Intervention an einem stark unterbestimmten Modell simulieren, dann wissen wir nicht, auf welchen Mechanismen (oder Wechselwirkungen zwischen Mechanismen) die Wirkung der Intervention basierte. Dies ist der Fall in Eubanks et al. [8]: Die Ergebnisse könnten von einigen oder allen der Mechanismen im Modell oder von ihren spezifischen Wechselwirkung abhängen, aber es ist unmöglich, diese Einflüsse auseinander zu halten. Mit ASMs lassen sich Analysen einfacher durchführen, weshalb sie für Entscheidungszwecke vorzuziehen sind.

\subsection{Strukturelle Unsicherheit}

Aus der bisherigen Diskussion folgt, dass Unsicherheit in der Modellspezifikation nie vollständig beseitigt werden kann, wie viel Details man auch immer in das Modell aufnehmen mag. Einige Unsicherheitsfaktoren betreffen MSMs mehr als ASMs, wie in den Abschnitten 3.2 und 3.3 beschrieben wird. Aber andere unvermeidbare Unsicherheiten ergeben sich aus der allgemeinen Fehlbarkeit des menschlichen Wissens und betreffen daher MSMs und ASMs gleichermaßen. In diesem Abschnitt werde ich die früheren differentiellen Probleme ignorieren und annehmen, dass MSMs und ASMs dem gleichen Grad an Unsicherheit ausgesetzt sind. Die Frage ist dann, ob MSMs und ASMs unterschiedliche Strategien anbieten, mit solchen unvermeidlichen Unsicherheiten umzugehen, und welche dieser Strategien besser ist.

Betrachten Sie das folgende Beispiel von Eubanks et al. [8]. Die Kontaktdaten, auf denen die Simulation basiert, geben eine detaillierte Darstellung der sozialen Interaktion. Das Modell enthält keine Angaben darüber, wie sich diese sozialen Kontakte unter externen Schocks verändern können. Die Ankunft einer bedrohlichen Epidemie ist wohl ein solcher Schock. Sie könnte einen wichtigen Einfluss darauf haben, wie oft Menschen in der Öffentlichkeit auftreten, zur Arbeit oder ins Krankenhaus gehen. Die Autoren beschäftigen sich mit dieser Unsicherheit wie folgt:

\footnotetext{
One of the most important assumptions in any smallpox model is whether infectious people are mixing normally in the population. [...] We undertook to model two (probably unrealistic) extreme cases: one in which no one who is infectious is mixing with the general population and another in which no one's behavior is affected at all by the disease. In addition, we modeled one more realistic case between these two extremes. ([8] Supplement:11)
} 
Die Modellergebnisse hängen stark von diesen unterschiedlichen Annahmen ab. Insbesondere wenn Menschen sich zu Hause zurückziehen, führt jede Impfpolitik zu ähnlichen Ergebnissen, insbesondere wenn sich das Impfverfahren verzögert. Wenn Menschen sich jedoch nicht zurückziehen, ist LV wesentlich weniger effektiv als entweder MV oder TV ([8]:182, Abb.4).

Beachten Sie, dass das MSM hier nur eine qualitative Unterscheidung erlaubt: Abhängig davon, ob der Entzug „früh“, „spät“ oder „nie“ erfolgt, führt die Simulation zu einer anderen kumulativen Anzahl von Todesfällen. Eine solche Analyse ist in ähnlicher Weise mit ASMs möglich. Die MSM-Autoren bewerten die in diesen qualitativen Ergebnissen enthaltenen Unsicherheiten nicht. Obwohl mir dies in diesem Fall das richtige Verfahren zu sein scheint - da nicht genügend Evidenz vorliegt, um eine quantifizierte Bewertung der Verhaltensänderungen unter Schocks zu liefern - stellt sich die Frage, warum man dann die zusätzlichen Anstrengungen und Kosten eines MSM auf sich nimmt, wenn ähnliche Ergebnisse mit einem ASM erreicht werden könnten.

Was MSM oft erreichen wollen, ist eine Gesamtquantifizierung der damit verbundenen Unsicherheit. Obwohl Eubanks et al. dies nicht anstreben (richtigweise, wie ich glaube), hätten sie versuchen können, eine Wahrscheinlichkeitsverteilung über die verschiedenen Verhaltensmechanismen zu spezifizieren und dann das Modellergebnis als erwartete kumulative Todesfälle darzustellen. Solche one-size-fits-all Ansätze in MSMs wurden zu Recht kritisiert, weil sie falsche Präzision liefern:

if uncertainty is represented and reported in terms of precise probabilities, while the scientist conducting the analysis believes that uncertainty is actually 'deeper' than this - e.g. believes that available information only warrants assigning wide interval probabilities or considering an outcome to be plausible - then the uncertainty report will fail to meet the faithfulness requirement; it will have false precision. ([25]:4)

Mein Argument hier ist, dass bei den meisten Anwendungen von MSMs für Entscheidungszwecke nicht quantifizierbare Unsicherheiten auftreten. Diese sollten nicht mit falscher Präzision überdeckt werden, wie im obigen Zitat beschrieben. Alternativ werden MSMs verwendet, um verschiedene qualitative Ergebnisse zu liefern, wie am Eubanks et al. Beispiel zu sehen - dies hätte aber auch von einem ASM geliefert werden können. Verteidiger von MSMs könnten hier antworten, dass solche qualitativen Ergebnisse von MSMs genauer sind als die Vergleichsergebnisse von ASMs. Meine früheren Ausführungen in den Abschnitten 3.2-3.4 stellen jedoch die Frage, ob dies notwendigerweise der Fall ist. Folglich sind die Strategien zur Unsicherheitsquantifizierung, die von MSMs unterstützt werden, nicht notwendigerweise besser als die Strategien von ASMs [26]. 


\section{Schlussfolgerungen}

In diesem Aufsatz argumentiere ich, dass MEMs oft für Entscheidungszwecke ausreichen. Sie isolieren einen zuverlässigen Mechanismus, mit dem der Entscheidungsträger das fragliche System systematisch beeinflussen und diese Interventionen von Beobachtungen des aktuellen Zustands abhängig machen kann. MEMs müssen nur dann ersetzt werden, wenn sie nicht ausreichend robust sind. Solche Robustheitsuntersuchungen erfordern jedoch nur die Verwendung von ASMs, nicht von MSMs. MSMs sind daher für politische Zwecke nicht notwendig.

MSMs, obwohl nicht notwendig, mögen für Entscheidungsträger dennoch als sehr bequem erscheinen. Gegen diesen Eindruck argumentierte ich, dass MSMs selten eine Qualität aufweisen, die der Entscheidung tatsächlich dienlich wäre. Insbesondere argumentierte ich, dass MSMs bei der Bestimmung der Genauigkeit des Modells schwerwiegendere Probleme haben können als ASMs. Dass MSMs im Umgang mit unvermeidlicher Unsicherheit größere Probleme haben können als ASMs; und schließlich, dass MSMs größere Probleme mit Fehlinterpretationen haben können.

Dies schließt natürlich nicht aus, dass einige MSMs gute Begründungen für Entscheidungen liefern (und sogar bessere Begründungen als einige ASMs). Mein Resultat, dass MSMs weder notwendig für rationale Entscheidungen sind, noch häufig Bedingungen erfüllen, die sie überhaupt zu guten Entscheidungshilfen qualifizieren, sollte Entscheidern Bedenken geben, MSMs allgemein ASMs als Entscheidungshilfe vorzuziehen.

\section{Literatur}

[1] Bicknell WJ. The case for voluntary smallpox vaccination. In: The New England journal of medicine. 2002;346(17):1323-1325.

[2] Hine D. The 2009 Influenza Pandemic: An independent review of the UK response to the 2009 influenza pandemic. 2010 (Available at https://www.gov.uk/government/uploads/system/ uploads/attachment_data/file/61252/the2009influenzapandemic-review.pdf [2.5.2016]).

[3] Vynnycky E, White R. An introduction to infectious disease modelling. Oxford University Press, 2010.

[4] Kaplan EH, Craft DL, Wein LM. Emergency response to a smallpox attack: The case for mass vaccination. In: Proceedings of the National Academy of Sciences. 2002;99(16):10935-10940.

[5] Kermack WO, McKendrick AG. A Contribution to the Mathematical Theory of Epidemics. In: Proceedings of the Royal Society. 1927;115(772):700-721.

[6] Halloran ME, Longini IM, Nizam A, Yang Y. Containing bioterrorist smallpox. In: Science. 2002;298(5597):1428-1432.

[7] Burke DS, Epstein JM, Cummings DA, et al. Individual-Based Computational Modeling Of Smallpox Epidemic Control Strategies. In: Academic Emergency Medicine. 2006;13(11):1142-1149.

[8] Eubank S, Guclu H, Kumar VSA, et al. Modelling Disease Outbreaks In Realistic Urban Social Networks. In: Nature. 2004;429:180-184 (see supplement at http://www.nature.com/nature/ journal/v429/n6988/extref/nature02541-s1.htm [2.10.2017]). 
[9] Farmer JD, Foley D. The economy needs agent-based modelling. In: Nature. 2009;460(7256):685-686.

[10] Dawid H, Fagolio G. Editorial. In: Journal of Economic Behaviour \& Organization. 2008;67:351-354.

[11] Kaplan EH. Preventing second-generation infections in a smallpox bioterror attack. In: Epidemiology. 2004;15(3):264-270.

[12] Cartwright N. Nature's Capacities and their Measurement. Oxford University Press, 1994.

[13] Mäki U. On the method of isolation in economics. In: Idealization IV: Intelligibility in science, ed. by Dilworth C. Amsterdam: Rodopi, 1992. 319-354.

[14] Kaplan EH, Craft DL, Wein LM. Analyzing bioterror response logistics: the case of smallpox. In: Mathematical Biosciences. 2003;185(1):33-72.

[15] Cartwright N. The vanity of rigour in economics: Theoretical models and Galilean experiments. In Hunting causes and using them: Approaches in philosophy and economics, ed. by Cartwright N. Cambridge University Press, 2007. 217-261.

[16] Grüne-Yanoff T. Isolation is Not Characteristic of Models. In: International Studies in the Philosophy of Science. 2011;25(2):1-19.

[17] Kuorikoski J, Lehtinen A, Marchionni C. Economic modelling as robustness analysis. In: The British Journal for the Philosophy of Science. 2010;61(3):541-567.

[18] Kaplan EH, Wein LM. Smallpox bioterror response. In: Science. 2003;300(5625):1503 .

[19] Halloran ME, Longini IM. Response. In: Ibidem. 1503-1504.

[20] Epstein, JM. Agent-based computational models and generative social science. In: Complexit. 1999;4(5):41-57.

[21] Myung IJ. The importance of complexity in model selection. In: Journal of Mathematical Psychology. 2000;44(1):190-204.

[22] Zucchini W. An introduction to model selection. In: Ibidem. 4-61.

[23] Gigerenzer G, Brighton H. Homo heuristicus: Why biased minds make better inferences. In: Topics in Cognitive Science. 2009;1(1):107-143.

[24] Lenhard J, Winsberg E. Holism, entrenchment, and the future of climate model pluralism. In: Studies in History and Philosophy of Science Part B: Studies in History and Philosophy of Modern Physics. 2010;41(3):253-262.

[25] Parker WS, Risbey JS. False Precision, Surprise and Improved Uncertainty Assessment. In: Philosophical Transactions of the Royal Society A: Mathematical, Physical and Engineering Sciences. 2015;373(2055):20140453.

[26] Grüne-Yanoff T. Choosing the Right Model For Policy Decision-Making: The Case of Smallpox Epidemiology. In: Synthese. 2018: 1-22 (https://doi.org/10.1007/s11229-018-1827-3). 